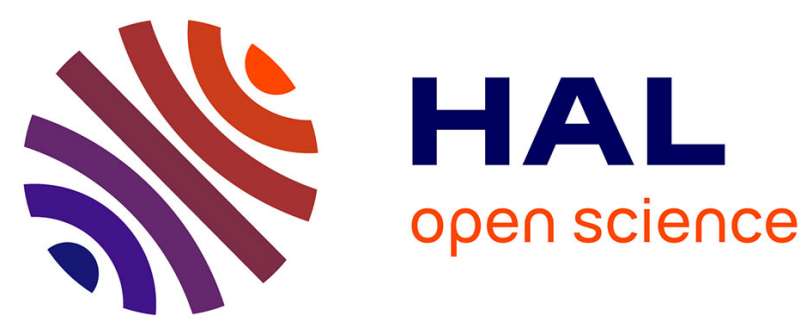

\title{
Lyapunov-Based Switching Control for a Road Estimation and Tracking Applied on a Convertible MAV
}

Gerardo Ramon Flores Colunga, Rogelio Lozano, Guillaume Sanahuja

\section{To cite this version:}

Gerardo Ramon Flores Colunga, Rogelio Lozano, Guillaume Sanahuja. Lyapunov-Based Switching Control for a Road Estimation and Tracking Applied on a Convertible MAV. AIAA Guidance, Navigation, and Control and Co-located Conferences and AIAA Infotech@Aerospace 2013, Aug 2013, Boston, Massachusetts, USA, United States. pp.1-13, 10.2514/6.2013-4940 . hal-00939197

\section{HAL Id: hal-00939197 https://hal.science/hal-00939197}

Submitted on 30 Jan 2014

HAL is a multi-disciplinary open access archive for the deposit and dissemination of scientific research documents, whether they are published or not. The documents may come from teaching and research institutions in France or abroad, or from public or private research centers.
L'archive ouverte pluridisciplinaire HAL, est destinée au dépôt et à la diffusion de documents scientifiques de niveau recherche, publiés ou non, émanant des établissements d'enseignement et de recherche français ou étrangers, des laboratoires publics ou privés. 


\title{
Lyapunov-Based Switching Control for a Road Estimation and Tracking Applied on a Convertible MAV
}

\author{
Gerardo Flores*, Rogelio Lozano ${ }^{\dagger}$ and Guillaume Sanahuja ${ }^{\ddagger}$ \\ Heudiasyc UMR 6599 Laboratory, University of Technology of Compiègne, France.
}

\begin{abstract}
This paper addresses the problem of estimation and tracking of a road using the new tiltrotor convertible MAV (mini aerial vehicle). For that objective, we consider the following scenario: (i) no previous knowledge of the road, i.e. shape, dimension and color, (ii) loss of information by the sensors is considered and (iii) nonlinear dynamics of the MAV is taken into consideration. Aiming at this goal, two operational regions are defined: road detected and road not detected by the sensors. A switching between the measurements of imaging and inertial sensors enables estimation of the required states in both operational regions. For dealing with both aforementioned cases, a Lyapunov-based switching control for stabilizing the vehicle's position is proposed. Unmodeled dynamics such as friction forces are estimated by means of the proposed controller. The global exponential stability of the position subsystem together with the switching controller is demonstrated exploiting the fact that the individual closed-loop systems are globally exponentially stable and the switching is sufficiently slow, so as to allow the transient effects to dissipate after each switch. The control law is validated on the Quad-plane experimental platform, ${ }^{1}$ showing the expected behavior during autonomous navigation.
\end{abstract}

\section{Introduction}

$\mathrm{T}$ HE rapid growth in electronics industry have permitted the development of electronic and sensor components like inertial measurement units, GPS, cameras and microcomputers. For this reason, in the last few years the control and robotics community have been interested in research control strategies to solve problems involving MAVs by exploiting such technologies.

Motivated by the need of developing effective MAV's controllers and the theory of switching systems, this research work deals not only with estimation problems, but also with performing a path following mission in presence of uncertainty parameters. In this paper, the main goal is getting the Quad-plane ${ }^{1}$ MAV to navigate autonomously and to follow a path represented by a highway model with the aim of tracking ground vehicles, exploring areas and monitoring highways. The road to be tracked is considered unknown, thus the vehicle must estimate the path in real-time. Furthermore, the mission must be performed in presence of external disturbances, which eventually will make the vehicle loses the highway. In order to deal with this situation, a state-dependent switching controller is proposed maintaining the MAV inside of the road. To achieve this goal, two operational regions are defined: road detected and road not detected. The first one describes the scenario where the MAV is positioned over the road, in a way that the vision sensor can capture the shape of the road. Otherwise, the MAV's position is outside of the road, an thus the objective must be restore the last position where the MAV is over the road. The stability of the closed-loop system on the individual regions is studied. Furthermore, the stability of the entire switching system is demonstrated by making use of some properties of the individual sub-systems.

\footnotetext{
*This work was partially supported by the Institute for Science \& Technology of Mexico City (ICyTDF). PhD candidate, Heudiasyc UMR 6599 Laboratory University of Technology of Compiègne, France. (email: gfloresc@hds.utc.fr).

${ }^{\dagger}$ CNRS Research Director, Heudiasyc UMR 6599 Laboratory, UTC CNRS France and LAFMIA UMI 3175, Cinvestav, México. (email: rlozano@hds.utc.fr)

$\ddagger$ Research associate, Heudiasyc UMR 6599 Laboratory University of Technology of Compiègne, France. (email: gsanahuj@hds.utc.fr).
} 


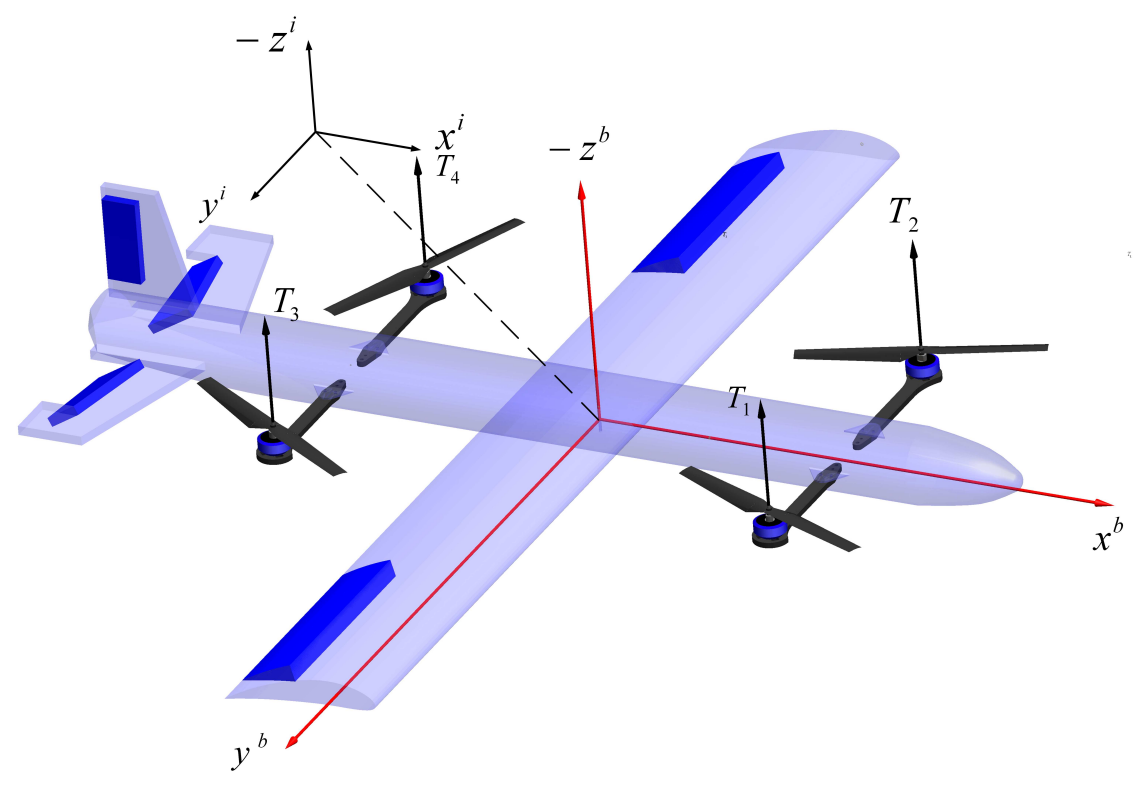

Figure 1. Quad-plane experimental platform.

The problem of stabilizing a MAV using visual feedback has been addressed by several research groups, see for example, ${ }^{2},{ }^{34}$ and the references therein. A proposed solution to handle the situation where the image is lost, is to implement different controllers, which is appropriate since the use of a PD controller in the position dynamics is not enough for attenuating disturbances. ${ }^{5}$ A switching strategy for estimating the states of an airplane equipped with imaging, inertial and air data sensing systems is introduced in. ${ }^{6}$ The proposed methodology demonstrates being capable of dealing with instants of time when the visual detection of the landmark fails. $\mathrm{In}^{7}$ a vision-based estimation algorithm is proposed to tracking a road. Also in, ${ }^{8}$ the authors present the adaptation of an optimal terrain coverage algorithm for the aerial robotics domain. The general strategy involves computing a trajectory through a known environment.

In this work, we will consider that uncertain parameters regarding unmodeled dynamics have to be estimated. To achieve this goal we implement a logic-based switching design strategy. Previous work on this subject can be seen in, ${ }^{9} .{ }^{10}$ The key idea to estimate the parameters is to split the set of possible parameters into smaller subsets, choose a controller for each parameter and switch to a different controller if the derivative of the Lyapunov function does not satisfy a certain inequality after a predefined time $\tau$.

The proposed control was tested in real-time experiments on the Quad-plane experimental platform, showing the expected behavior during the navigation task. It is important to say, that all the vision and control algorithms are embedded on the Quad-plane MAV.

The body of the papers is as follows. In section II the problem statement is introduced. Next, in section III the model of the vehicle in hover mode is presented. After that, the methods for estimating the states of the Quad-plane are shown in Section IV. Section V presents a switching control strategy as well as the stability proof. Section VI presents the Quad-plane experimental platform. The behavior of the Quadplane during real-time experiments is shown in Section VII. Finally, some conclusions and future works are presented in section VIII.

\section{Problem Statement}

The road following procedure can be detailed as follows. First, the vehicle performs an autonomous take-off, reaching a desired altitude $z_{d}$ over the road. Then, the heading of the vehicle $\psi$, is controlled to obtain a parallel positioning between the Quad-plane $x$-axis $x_{h}$ and the longitudinal direction of the highway $x_{r}$ as shown by figure 3 on page 4 .

The Quad-plane forward velocity $\dot{x}$ is maintained constant while the distance between $x_{h}$ and $x_{r}$, expressed by $e_{y}$, is controlled and maintained to a minimum value, achieving a flight path well aligned and 


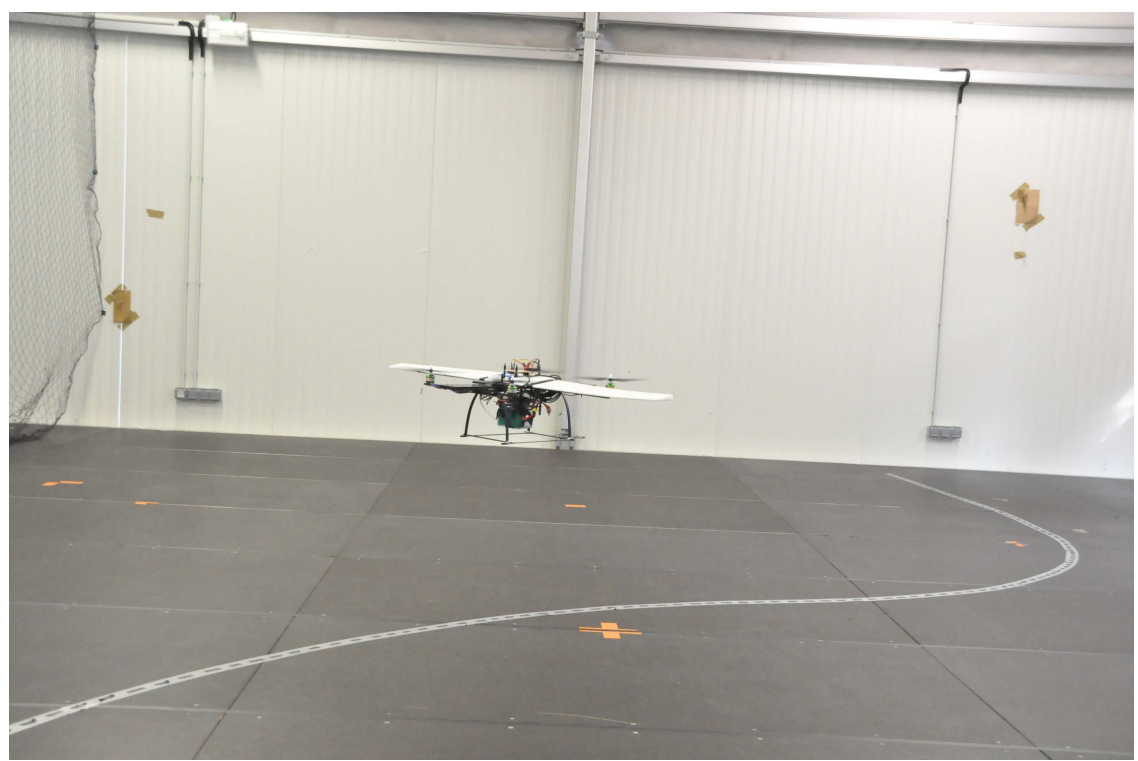

Figure 2. Experimental platform tracking the road.

centered w.r.t. the highway at constant forward velocity. Finally the Quad-plane performs a landing in a position near to the end of the highway. All of these actions should be accomplished completely autonomously without the previous knowledge of shape of the highway and the terrain.

During some instants of time, the road that is being tracked will not be detected in the camera's image, due to occlusions or image blurring, but this can also occur when the vehicle experiences external disturbances causing the Quad-plane to lose its stability and the navigation path. In the times when the highway is out of the camera field of view, the Quad-plane heading angle is controlled from the $\psi$-angle measurement that the IMU provides. A pointing downwards camera allows to estimate the vehicle's heading angle with respect to the road's longitudinal orientation, as well as the lateral distance that must be corrected in order to navigate exactly over the road.

Two operational regions are defined with the purpose of developing a switching control strategy for estimation and tracking: the first one concerns the situation when the road is detected, while the second one deals when it is not. It must be shown that inside the aforementioned regions, the system is globally exponentially stable (GES). In addition it must be proved that the entire closed-loop system is GES even in the switching boundaries, i.e. at the times where a switching signal between both regions occurs.

Moreover, in order to improve the stability response, the vehicle has to estimate the parameter corresponding to the friction force presented on the mathematical model. To carry out this objective, a series of controllers provided by one switching algorithm, are chosen according to a certain condition on the corresponding Lyapunov function and a given time $\tau$. The objective is to accomplish stability through switching between several candidate controllers to achieve satisfactory performance for a subset of parameter uncertainty, ${ }^{10} \cdot 9$

\section{Modeling}

In this work, we will focus on the hover mode of the Quad-plane MAV. In this mode, our vehicle behaves like a conventional Quad-rotor, then we will use the Quad-rotor mathematical model. ${ }^{11}$

$$
\begin{aligned}
m \ddot{x} & =u_{1}(\sin \psi \sin \phi+\cos \psi \sin \theta \cos \phi)-\frac{1}{2} C_{x} A_{c} \rho \dot{x}|\dot{x}| \\
m \ddot{y} & =u_{1}(\sin \psi \sin \theta \cos \phi-\cos \psi \sin \phi)-\frac{1}{2} C_{y} A_{c} \rho \dot{y}|\dot{y}| \\
m \ddot{z} & =m g-u_{1}(\cos \theta \cos \phi) \\
\ddot{\theta} & =u_{2} \\
\ddot{\phi} & =u_{3} \\
\ddot{\psi} & =u_{4}
\end{aligned}
$$


where $u_{i}$ are the control inputs, $(x, y, z)$ are the position states and $(\theta, \phi, \psi)$ are the orientation angles. $C_{x}$, $C_{y}, A_{c}$ and $\rho$ are unknown parameters related to the friction force. ${ }^{12}$ The corresponding coordinate system is represented in figure 1 on page 2 . The following set of state variables are used:

$$
\begin{array}{llllll}
x_{1}=x & y_{1}=y & z_{1}=z & \theta_{1}=\theta & \phi_{1}=\phi & \psi_{1}=\psi \\
x_{2}=\dot{x} & y_{2}=\dot{y} & z_{2}=\dot{z} & \theta_{2}=\dot{\theta} & \phi_{2}=\dot{\phi} & \psi_{2}=\dot{\psi}
\end{array}
$$

\section{States estimation}

A sufficiently smooth road is represented in the camera's image as a group of lines, (Fig. 3). A straight line in the image can be seen as a segment of infinite length and whose center of gravity belongs to the straight line. ${ }^{13}$ The $\psi_{d}$ angle can be represented as

$$
\psi_{d}=\psi_{r}+\frac{\pi}{2}
$$

The details of how we estimated the $\psi_{d}$ angle shown in Fig. 3, relative position and velocity with respect to the road, can be seen in our previews work. ${ }^{14}$
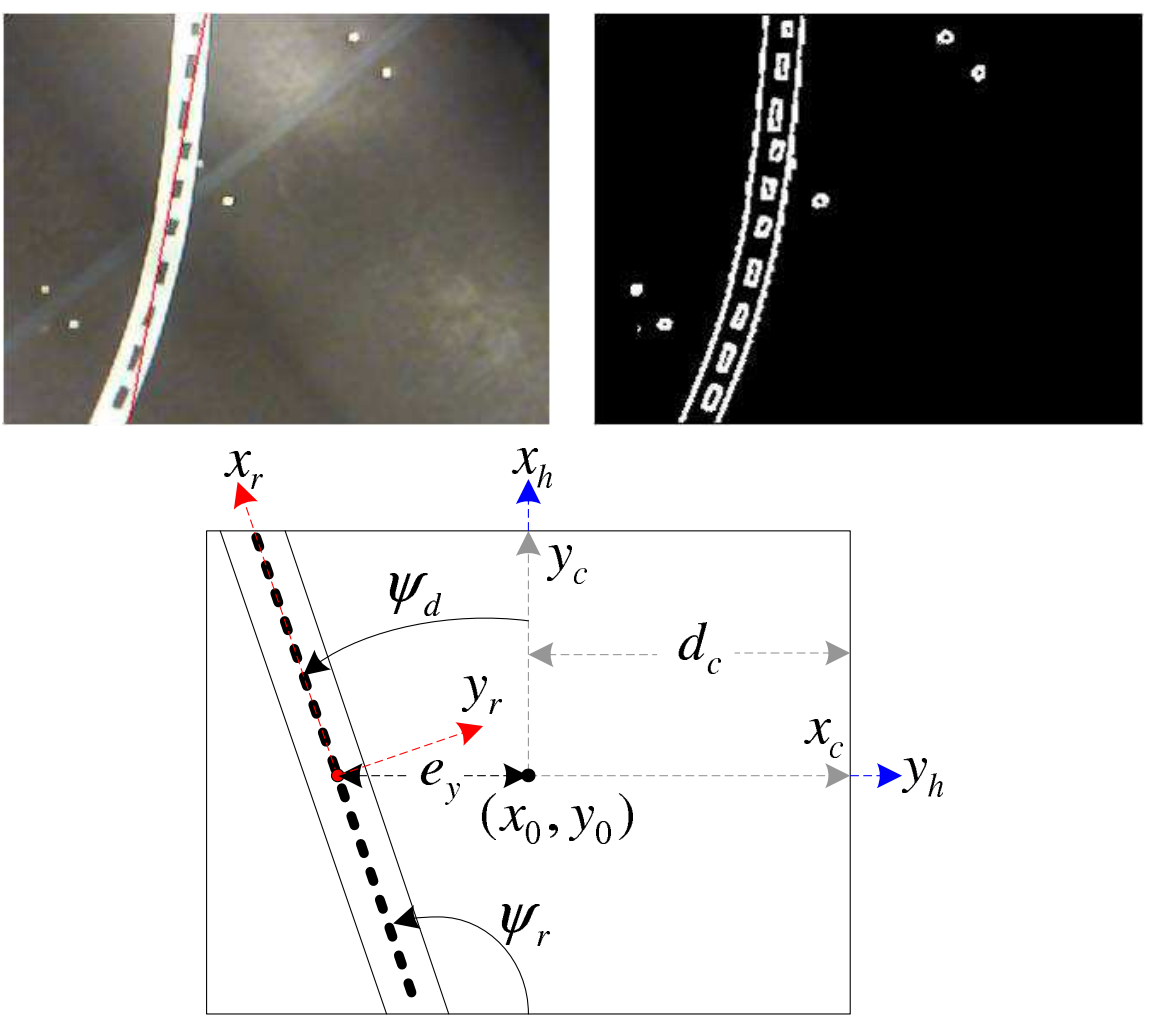

Figure 3. Schema of the camera's view, showing the captured image.

\section{IV.A. States computation when the road is out of the camera's field of view}

In a real experimental application, the image of the road can be lost in certain instants of time. To address this problem, let's define a binary signal $s:[0, \infty) \rightarrow\{0,1\}$ as

$$
s(t):= \begin{cases}0 & \text { road not detected at time } t \\ 1 & \text { road detected at time } t\end{cases}
$$

which allows to switch between two different strategies for computing the vehicle's states. We define also the time event $T_{s 0}$ as the time when the binary signal switches from 1 to 0 , and $T_{s 1}$ the time event when the binary signal switches from 0 to 1. 
Consider the desired heading angle computation. The equation (3) permits to define

$$
\psi_{s}(t)=s(t) \psi_{d}(t)+(1-s(t)) \psi_{I M U}\left(T_{s 0}\right)
$$

where $\psi_{d}(t)$ is obtained from (2), $\psi_{I M U}\left(T_{s 0}\right)$ is the IMU's heading angle measurement at $T_{s 0}$, and $\psi_{s}(t)$ is the desired heading angle that will be used in the Quad-plane embedded controller. From this result, (4) allows switching from a $\psi_{s}(t)$ generated by the imaging algorithm, and a $\psi_{s}(t)$ defined by the IMU's heading angle value at the time when the road was not detected on the image.

Now, lets consider the vehicle's lateral position estimation when the road is out of the camera's field of view. Equation (3) permits to estimate

$$
e_{y s}(t)=s(t) e_{y}(t)+(1-s(t))\left(e_{y}\left(T_{s 0}\right)+\int_{T_{s 0}}^{T_{s 1}} \dot{y}(t) d(t)\right)
$$

where $e_{y}(t)$ is a vision-based estimated, $e_{y}\left(T_{s 0}\right)$ is the lateral position estimation at $T_{s 0}$, and $\dot{y}(t)$ is the vehicle's lateral velocity obtained from optical flow estimation. From the equation (5), it is possible to switch between two different methods for estimating the vehicle's lateral position w.r.t. the road.

In the first method, the lateral position is obtained from the road line detection in the camera's image. The second method uses the road's position at $T_{s 0}$ in combination with the integral of $\dot{y}$ during the time the road is not being detected by the imaging algorithm, i.e., during a time defined by time events $T_{s 0}$ and $T_{s 1}$.

Remark 1: It is important to mention that a similar procedure is performed for the $x$ dynamics of the MAV.

\section{Control Strategy}

The proposed control strategy is based on the idea that the global system (1) is constituted of two subsystems, the attitude dynamics and the position dynamics, each one with a time-scale separation between them. ${ }^{4}$ From this fact, it is possible to propose a hierarchical control scheme where the position controller outputs desired attitude angles $\phi_{d}, \theta_{d}, \psi_{d}$ ( $\psi_{d}$ can be independently controlled) which are the angles to be tracked by the orientation controllers.

\section{V.A. Attitude Control}

The integral sliding mode control is used for stabilizing the attitude dynamics of the Quad-plane. The approach is explained for the roll dynamics, but the same procedure must be followed for generating the pitch and yaw dynamics. The error equation for the roll sub-system is defined as $\tilde{\phi}_{1}=\phi_{1}-\phi_{1_{d}}$, and its time derivative as $\dot{\tilde{\phi}}_{1}=\dot{\phi}_{1}-\dot{\phi}_{1_{d}}=\tilde{\phi}_{2}$. Lets choose the switching function defined in ${ }^{15}$ as

$$
\varphi(\phi, t)=\dot{\tilde{\phi}}_{1}+2 \lambda \tilde{\phi}_{1}+\lambda^{2} \int_{0}^{t} \tilde{\phi}_{1}(\tau) \mathrm{d} \tau
$$

In (6) the parameter $\lambda$ is the slope of the sliding line, which should accomplished $\lambda>0$ to ensure the asymptotic stability of the sliding mode. The time derivative of (6) can be calculated as $\dot{\varphi}=u_{3}+2 \lambda \tilde{\phi}_{2}+\lambda^{2} \tilde{\phi}_{1}$, and from the sliding mode condition $\dot{\varphi}=0$, we find the equivalent control

$$
u_{3}=-2 \lambda \tilde{\phi}_{2}-\lambda^{2} \tilde{\phi}_{1}
$$

For obtaining a control law such that $\tilde{\phi}_{1}$ remains on the sliding surface $\varphi(\phi, t)=0, \forall t>0$, a candidate Lyapunov function $v(\varphi)=\frac{1}{2} \varphi^{2}$ is proposed. The condition for the stability of the roll sub-system is satisfied if we can ensure that the condition $\dot{v}(\varphi)=\frac{1}{2} \frac{d}{d t} \varphi^{2} \leq \eta|\varphi|$ holds for $\eta \geq 0$. Thus, the system remains on the sliding surface and the states go to the origin. Then $\varphi \dot{\varphi} \leq-\eta|\varphi|$ and the controller must be chosen in a way that $\phi_{1}=u_{3}-K \operatorname{sign}(\varphi)$ where $K>0$.

\section{V.B. Position Control}

The lateral position control is formed by a pair of PID controllers, one for the case where the highway is detected and the other one for the case where it is not. The PID controllers have different gains with the goal of obtaining a behavior like a PD (when the highway is inside of the camera's field of view) and a PI (when 
the highway is not detected) controller according to the gain values chosen. The PD controller ensures a rapid response for maintaining the vehicle's $y$ coordinate at a minimum value, i.e., $y=0 .^{5}$ The PI controller is used when the vehicle looses the image of the highway, since at this moment a switch to an alternative method for measuring the $\psi$ angle and $y$ position occurs. In both cases the control objective is to regulate the $y_{1}$ state to the origin, i.e. $y_{1 d}=0$. Thus, we proceed to develop a control law for the $y$-position.

In (1) we can note that the motion along $y$-axis is related to the $\phi$ angle, thus one can design a PID controller to manipulate the $\phi$ angle in order to control $y$ motions. Setting $\theta=\psi=0$ and $u_{1}=1$ from (1) the term $\ddot{y}$ results in

$$
\ddot{y}=-u_{1} \sin \phi-p \dot{y}^{2}=-k_{L 3} y_{1}-k_{L 4} y_{2}-k_{L I} \xi
$$

where $p \dot{y}^{2}=\frac{1}{2} C_{y} A_{c} \rho \dot{y}|\dot{y}|$ and $p$ is a unknown parameter to be estimated; $k_{L 3}, k_{L 4}$ and $k_{L I}$ are positive real numbers. Here we have introduced the additional state $\xi$, where $\xi$ dynamics are given by $\dot{\xi}=y_{1 d}-y_{1}=-y_{1}$.

From (8) the desired angle $\phi_{d}$ can be written as

$$
\phi_{d}=\arcsin \left(k_{L 3} y_{1}+k_{L 4} y_{2}+k_{L I} \xi-p \dot{y}^{2}\right)
$$

By taking the derivative of (9) one obtains

$$
\dot{\phi}_{d}=\frac{k_{L 3} \dot{y}+k_{L 4} \ddot{y}+k_{L I} \dot{\xi}-2 p \dot{y} \ddot{y}}{\sqrt{1-v}}
$$

where $v$ is a function of $(p, y, \dot{y})$.

Proceeding in the same way one can obtain $\theta_{d}$ and its time derivative $\dot{\theta}_{d}$ required for the controller $u_{2}$, which can be generated with the procedure shown in section V.A.

The altitude, can be controlled by the following controller

$$
u_{1}=\frac{g+k_{p z}\left(z_{1}-z_{1_{d}}\right)+k_{v z}\left(z_{2}-z_{2_{d}}\right)}{\cos \theta \cos \phi}
$$

where $k_{p z}$ and $k_{v z}$ are positive real numbers.

\section{V.B.1. Discontinuous Adaptation for y Dynamics}

In order to apply the virtual control law (9) on the dynamics of $y$, we need to estimate the unknown parameter $p$. To achieve this goal, the key idea is to split the set of parameters into small subsets and design a controller for each of them. Then, after a dwell-time period on needs to check if the derivative of the Lyapunov function does not satisfy a certain inequality, if this is the case, one needs to switch to another controller and exclude the previous one from the indexed controls. As long as the inequality is satisfied, the current controller is maintained in the loop. The procedure is explained as follows.

We assume that the parameter $p$ belongs to a known, relatively large, compact set $\mathcal{P}$, which is partitioned into smaller subsets. Defining the compact set $\mathcal{P}$ as

$$
p \in \mathcal{P}=\bigcup_{i=1}^{21} p^{(i)}=\{-1,-0.9,-0.8, \ldots, 0.8,0.9,1\}
$$

With the controller previously designed (9), the closed-loop system (8), (9) leads to

$$
\begin{aligned}
\dot{y}_{1} & =y_{2} \\
\dot{y}_{2} & =-k_{L 3} y_{1}-k_{L 4} y_{2}-k_{L I} \xi \\
\dot{\xi} & =-y_{1}
\end{aligned}
$$

Considering $k_{L I}=0$, or equivalently, the case where we use a PD controller in (8), the Lyapunov function for the system (13), can be taken as

$$
V\left(y_{1}, y_{2}\right)=\sqrt{k_{L 3}}\left(1+\frac{k_{L 4}}{2 \sqrt{k_{L 3}}}\right) y_{1}^{2}+y_{1} y_{2}+\frac{y_{2}^{2}}{\sqrt{k_{L 3}}}
$$


whose derivative with respect to (8) is given by

$$
\dot{V}\left(y_{1}, y_{2}\right)=-k_{L 3} y_{1}^{2}-\left(\frac{2 k_{L 4}}{\sqrt{k_{L 3}}}-1\right) y_{2}^{2}=-W\left(y_{1}, y_{2}\right)
$$

where $\frac{2 k_{L 4}}{\sqrt{k_{L 3}}}>1$.

To choose the correct candidate controller or equivalently, the next suitable index $i \in \mathcal{I}$, we take such index as

$$
i=\arg \min _{j \in \mathcal{I}}\left\{J\left(p^{(j)}\right)\right\}
$$

where the performance index can be taken as

$$
J\left(p^{(j)}\right)=\left|\dot{y}_{2}+\sin \phi+p^{(j)} y_{2}^{2}\right|
$$

For each set $\mathcal{P}^{j}$ we choose a nominal parameter (for example 1) and assuming that the sets $\mathcal{P}^{j}$ are small, it is reasonable to expect $\left|J(p)-J\left(p^{(j)}\right)\right|$ to be small $\forall p \in \mathcal{P}^{j}$, therefore we use $J\left(p^{(j)}\right)$ as an index for the set $\mathcal{P}^{j}$. Thus we proceed with $i=1$, and we put the first candidate virtual controller (9) as follows

$$
\phi_{d}=\operatorname{Sat}\left(\arcsin \left(k_{L 3} y_{1}+k_{L 4} y_{2}-p^{(i)} \dot{y}^{2}\right)\right)
$$

where $\operatorname{Sat}(\cdot)$ is a smooth saturation function which switch to another one as soon as the dwell-time period $\tau$ is over and the inequality

$$
\frac{\partial V\left(y_{1}, y_{2}\right)}{\partial y_{1}} y_{2}+\frac{\partial V\left(y_{1}, y_{2}\right)}{\partial y_{2}} \dot{y}_{2} \leq-W\left(y_{1}, y_{2}\right)
$$

fails.

\section{V.B.2. Switching control for y-dynamics}

In this section we prove the stability in each region as well as in switching boundaries, i.e., when the gains of (8) switch to alternative values. The closed-loop system of the $y$ dynamics (13) can be represented by $\dot{e}_{y}=A_{L} e_{y}$ where $e_{y}=\left(y_{1}, y_{2}, \xi\right)^{T}$ and

$$
A_{L}=\left(\begin{array}{ccc}
0 & 1 & 0 \\
-k_{L 3} & -k_{L 4} & k_{L I} \\
-1 & 0 & 0
\end{array}\right)
$$

The control schema when the line is not detected proposes the same structure given by (13), with the difference of having a set of alternative gains. Thus, the closed-loop system is represented by $\dot{e}_{y}=A_{N L} e_{y}$, where

$$
A_{N L}=\left(\begin{array}{ccc}
0 & 1 & 0 \\
-k_{N L 3} & -k_{N L 4} & k_{N L I} \\
-1 & 0 & 0
\end{array}\right)
$$

It is important to mention that in the experimental platform, the gains were chosen experimentally, this resulted in different values for both cases. When the road is detected, the values were selected as $K_{L 3}=1800$, $K_{L 4}=650$ and $K_{L I}=1$. Gains were selected as $k_{N L 3}=1800, k_{N L 4}=1$ and $k_{N L I}=20$ for the case where the highway is not detected.

\section{V.B.3. Stability Analysis of the Lateral Position Control}

We now present a study concerning the stability of the system across switching boundaries. In fact, it is possible to find a common Lyapunov function for the closed-loop system of the two controllers proposed for the lateral dynamics. ${ }^{16}$ Following such approach, similar pole locations have to be chosen for both cases, i.e., when the road is detected and when it is not. However, this is not the present situation since different gain values are being applied. 
Let $d_{\mathrm{c}}$ be defined as the distance measured from the vehicle center of gravity projection (in the road plane) to the point where the camera loses the image of the road (see Figure 3). From this, a statedependent switched linear system can be defined, which will be given by the closed-loop system (20)-(21), together with the following switching conditions

$$
\dot{e}_{y}= \begin{cases}A_{N L} e_{y} & \text { if } y_{1} \leq-d_{\mathrm{c}} \\ A_{L} e_{y} & \text { if }-d_{\mathrm{c}}<y_{1}<d_{\mathrm{c}} \\ A_{N L} e_{y} & \text { if } y_{1} \geq d_{\mathrm{c}}\end{cases}
$$

A switching event can occur in system (22) every time the trajectory crosses a switching surface. For the case under consideration, the switching surfaces are encountered when the system changes from $\dot{e}_{y}=A_{L} e_{y}$ to $\dot{e}_{y}=A_{N L} e_{y}$, and viceversa. In general, there is no need to associate each subsystem in (22) with a global Lyapunov function. In fact, it is enough to require that each candidate Lyapunov function $V_{i}$ (where $i=\{L, N L\})$ decreases along solutions of the $i$-th subsystem in the region where this system is active.

The stability of the overall system (22) is accomplished when: (i) individual subsystems are stable, and (ii) the overall system remains stable when a switching surface is crossed. Indeed, it is possible to verify that individual subsystems of the switching system (22) are globally exponentially stable. Furthermore, if the switching between individual subsystems is sufficiently slow, the entire system (22) will be globally exponentially stable. ${ }^{17}$ Aiming at this goal, let us define a dwell time $\tau_{d}$ satisfying $t_{n+1}-t_{n} \geq \tau_{d}$, where $t_{n}$ (for $n=1,2,3, \ldots$ ) represents a switching time. Next, it is required to investigate the lower bound on $\tau_{d}$ from the exponential decay bounds of the individual subsystems. This procedure is explained as follows.

As previously mentioned, individual subsystems are GES, then there exist Lyapunov functions $V_{L}, V_{N L}$ which, for some positive constants $a_{i}, b_{i}$, and $c_{i}$ satisfy ${ }^{18}$

$$
a_{i}\left\|e_{y}\right\|^{2} \leq V_{i}\left(e_{y}\right) \leq b_{i}\left\|e_{y}\right\|^{2}
$$

and

$$
\frac{\partial V_{i}}{\partial e_{y}} A_{i} e_{y} \leq-c_{i}\left\|e_{y}\right\|^{2}
$$

for $i=\{L, N L\}$. Combining (23) and (24) we have

$$
\frac{\partial V_{i}}{\partial e_{y}} A_{i} e_{y} \leq-\frac{c_{i}}{b_{i}} V_{i}\left(e_{y}\right)
$$

This implies that

$$
V_{i}\left(e_{y}\left(t_{0}+\tau_{d}\right)\right) \leq e^{-\frac{c_{i}}{b_{i}} \tau_{d}} V_{i}\left(e_{y}\left(t_{0}\right)\right)
$$

Let us consider two switching times $\left\{t_{1}, t_{2}\right\}$, and let us also assume that subsystem $\dot{e}_{y}=A_{L} e_{y}$ is active on the interval $t \in\left[t_{0}, t_{1}\right)$, while subsystem $\dot{e}_{y}=A_{N L} e_{y}$ is active on $t \in\left[t_{1}, t_{2}\right)$. From inequalities (23)-(26) it follows that

$$
V_{L}\left(t_{2}\right) \leq \frac{b_{L}}{a_{N L}} V_{N L}\left(t_{2}\right) \leq \frac{b_{L}}{a_{N L}} e^{-\frac{c_{N L}}{b_{N L}} \tau_{d}} V_{N L}\left(t_{1}\right)
$$

and also

$$
V_{L}\left(t_{2}\right) \leq \frac{b_{N L} b_{L}}{a_{L} a_{N L}} e^{-\left(\frac{c_{L}}{b_{L}}+\frac{c_{N L}}{b_{N L}}\right) \tau_{d}} V_{L}\left(t_{0}\right)
$$

In order to find the lower bound on $\tau_{d}$ it is sufficient to ensure that (see Theorem 1 of $^{19}$ )

$$
V_{L}\left(t_{2}\right)-V_{L}\left(t_{0}\right) \leq-\sigma\left\|e_{y}\left(t_{0}\right)\right\|^{2}
$$

where $\sigma>0$. It is now straightforward to compute an explicit lower bound on $\tau_{d}$, which guarantees that the switched system $(22)$ is $\operatorname{GES}^{16}$

$$
\tau_{d}>\frac{b_{L}+b_{N L}}{c_{L}+c_{N L}} \log \frac{b_{L} b_{N L}}{a_{L} a_{N L}}
$$

The lower bound on $\tau_{d}$ can be satisfied by adjusting $a_{i}, b_{i}$, and $c_{i}$, for $i=\{L, N L\}$, which depend on the controller gains and the Lyapunov function. 


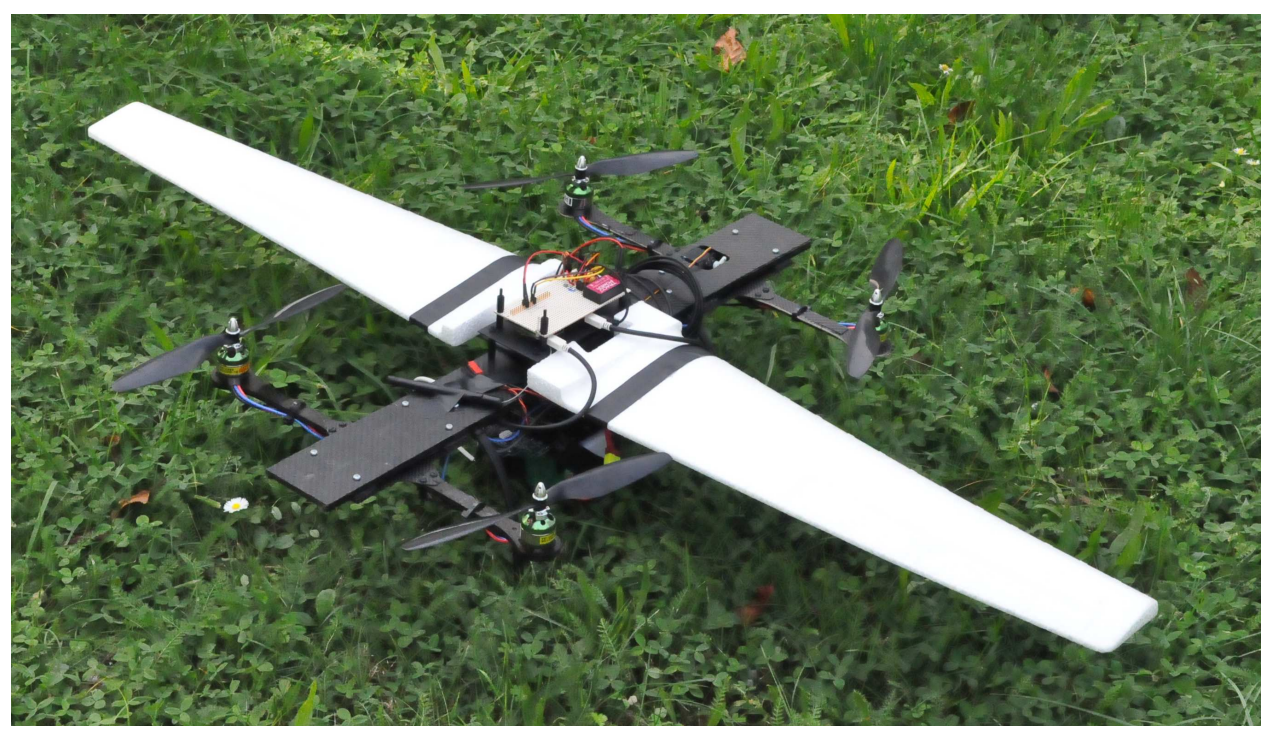

Figure 4. Quad-plane platform.

\section{Experimental platform}

\section{VI.A. Quad-plane platform}

The proposed controller has been tested on the Quad-Plane experimental platform illustrated in Fig. 4. The control algorithm is implemented on the the Gumstix computer that is based on a $720 \mathrm{MHz}$ ARM Cortex-A8 CPU with 512MB flash memory. It is the heart of the embedded system and constitutes the Flight Control Computer (FCC). The control law is executed in real-time at a $100 \mathrm{~Hz}$ frequency. Inertial measurements are provided at $100 \mathrm{~Hz}$ by means of a 3DMGX3-25 Inertial Measurement Unit (IMU) from Microstrain ${ }^{\circledR}$. For more details of the experimental platform see. ${ }^{3}$

\section{VI.B. Embedded vision system}

The embedded vision system includes a PlayStation ${ }^{\circledR}$ Eye camera, and a Flying Netbook computer, where the vision algorithms are programmed. The camera is capable of providing 120 images per second of a $320 \times 240$ resolution. The camera is used for observing the scene below the vehicle and record the image and video. The translational velocity in the $x-y$ plane is obtained from an optical flow algorithm, which is based on the pyramidal Lucas-Kanade method.

\section{VI.C. Ground station}

The helicopter is communicated wireless to a ground station PC where a graphical user interface (GUI) allows monitoring and controlling the Quad-plane. The user can visualize, in real-time, graphics representing the measurements from the on-board sensors as well as graphics representing the control law computation.

\section{Experimental Results}

The strategies proposed in Section V, were validated in real-time experiments, which can be described as follows. An autonomous take-off is performed, where the goal is to achieve a desired altitude of $0.70 \mathrm{~m}$ over the road. Once at this height, the helicopter can detect the road, which allows orientating its heading and stabilizing its lateral position, achieving a flying path well centered over the road. The vehicle is then required to navigate forward at a desired speed given by the user, regulating its heading angle and lateral position w.r.t. the road. Once the vehicle is near to the end of the road segment, the landing is performed autonomously, and the vehicle descends slowly to the ground.

The switching between the two states estimation approaches is illustrated in figure 8 on page 12 . The graphic on the middle represents the time instants where the line is being detected $(s=1)$ and where the 

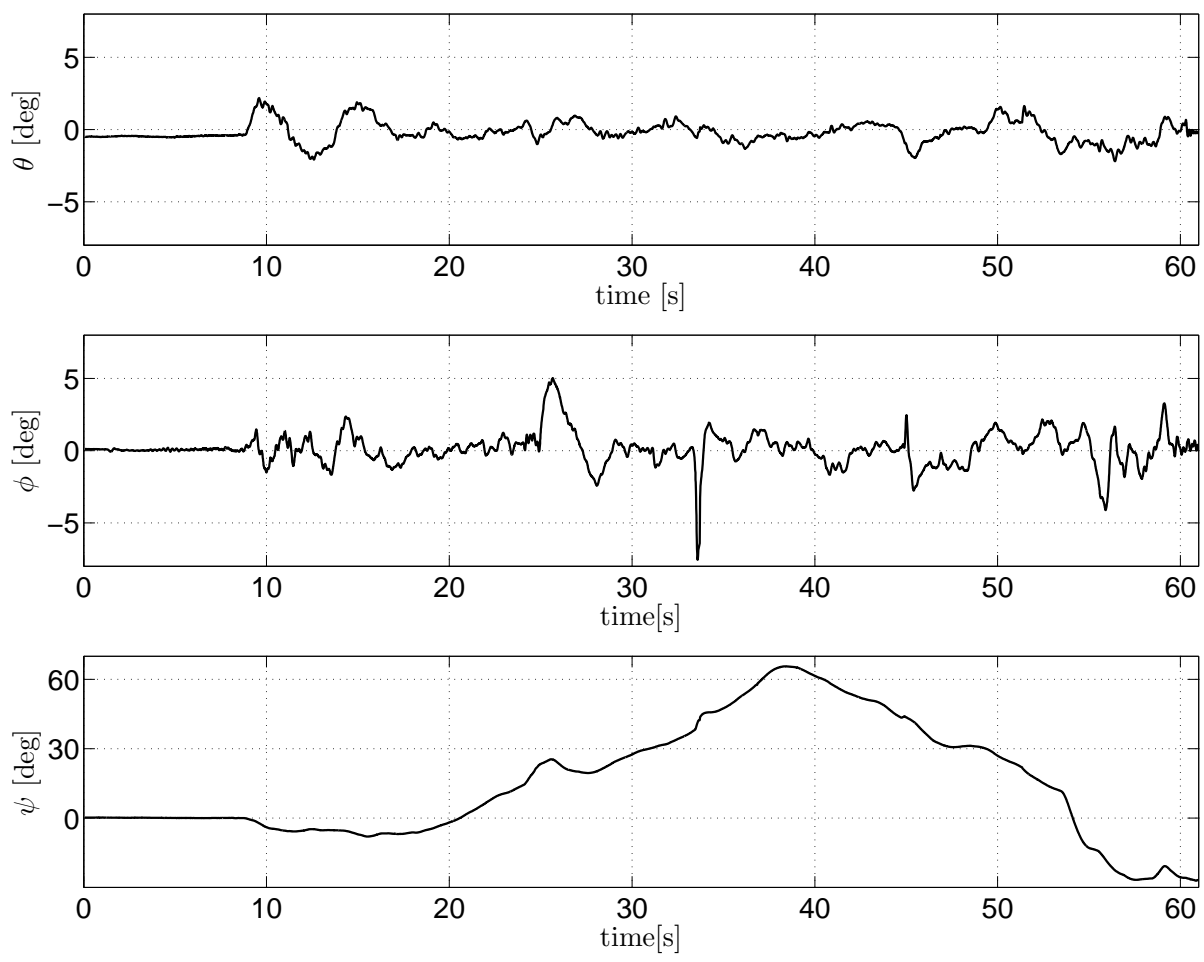

Figure 5. Behavior of the Euler angles during the experiment. The system was disturbed in the $\psi$ angle at $t=34$ s.

line is not being detected $(s=0)$. The upper graphic represents the desired heading angle as computed from (4). The lower graphic represents the lateral position error as computed from (5). As we can see in the figures 5, 6 on the next page, 7 on the following page, 8 on page 12 and 9 on page 12, the vehicle was perturbed in $\psi$ angle and in the $y$ position.

\section{Concluding Remarks}

The proposed estimating and control strategies were evaluated on the Quad-plane experimental platform during a highway following task, obtaining satisfactory results and demonstrating that the control proposed is effective in the presence of external disturbances.

All algorithms are optimized to run on standard computer hardware onboard the Quad-plane and provide key enabling techniques for its autonomous navigation.

The GES of the entire system is demonstrated by means of the exponential decay bounds of the individual subsystems.

Future work will focus on the development of a hybrid control for switching not only controller gains but also control strategies.

\section{References}

${ }^{1}$ Flores, G., Escareno, J., Lozano, R., and Salazar, S., "Quad-Tilting Rotor Convertible MAV: Modeling and Real-Time Hover Flight Control," Journal of Intelligent and Robotic Systems, Vol. 65, No. 1-4, 2012, pp. 457-471.

${ }^{2}$ Altug, E., Ostrowski, J., and Mahony, R., "Control of a quadrotor helicopter using visual feedback," International Conference on Robotics and Automation, Washington, DC, May, 2002, pp. 72-77.

${ }^{3}$ Zingg, S., Scaramuzza, D., Weiss, S., and Siegwart, R., "MAV Navigation through Indoor Corridors Using Optical Flow," International Conference on Robotics and Automation, Anchorage, Alaska, 2010, pp. 3361-3368.

${ }^{4}$ Flores, G. and Lozano, R., "Lyapunov-based Controller using Singular Perturbation Theory: An Application on a miniUAV," Proc. IEEE American Control Conference (ACC'2013), Washington, DC, June 2013, pp. 1599-1604. 

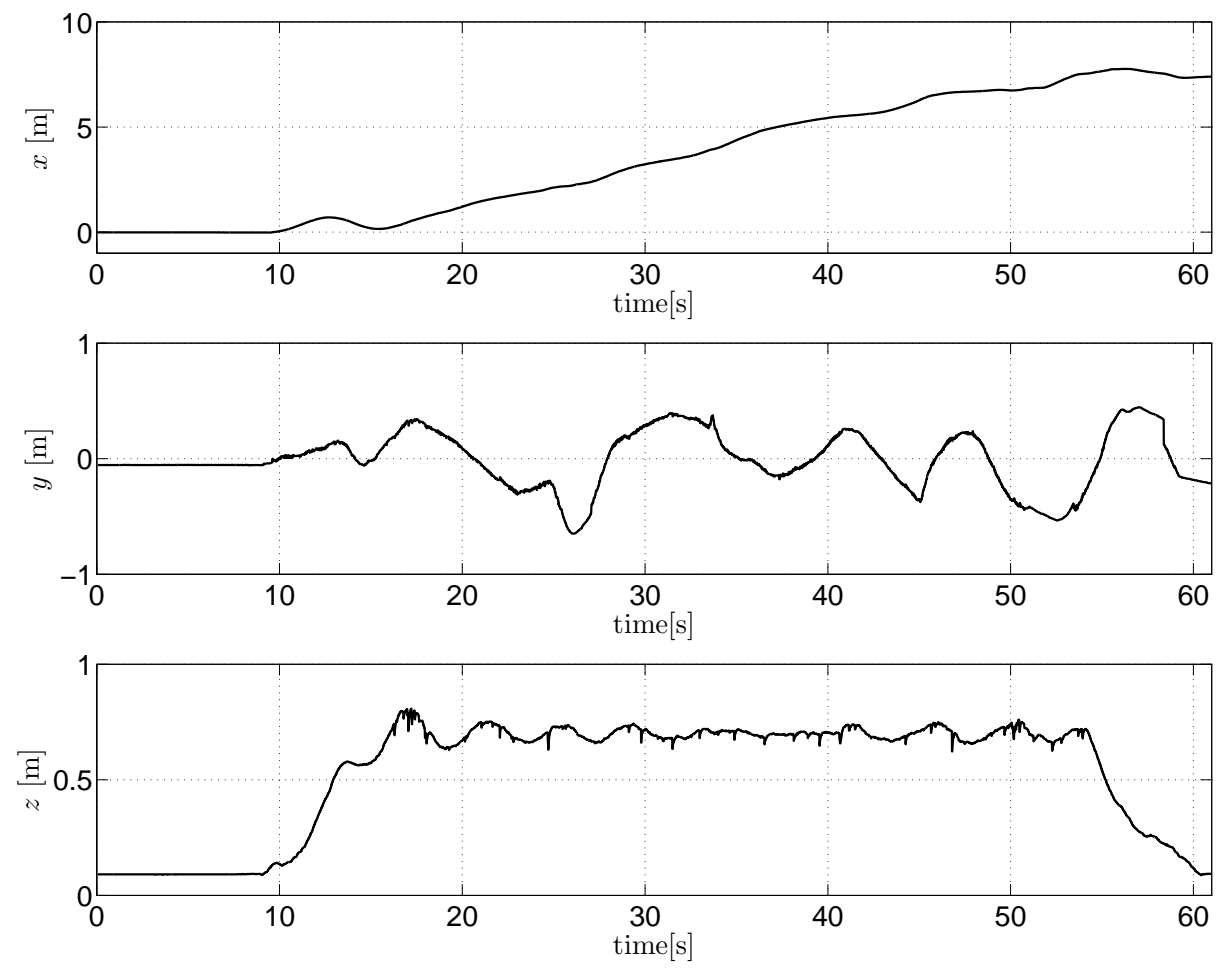

Figure 6. Position of the vehicle during the experiment. The system was disturbed in the $y$ position at $t=26 \mathrm{~s}$ and $t=45$ s.
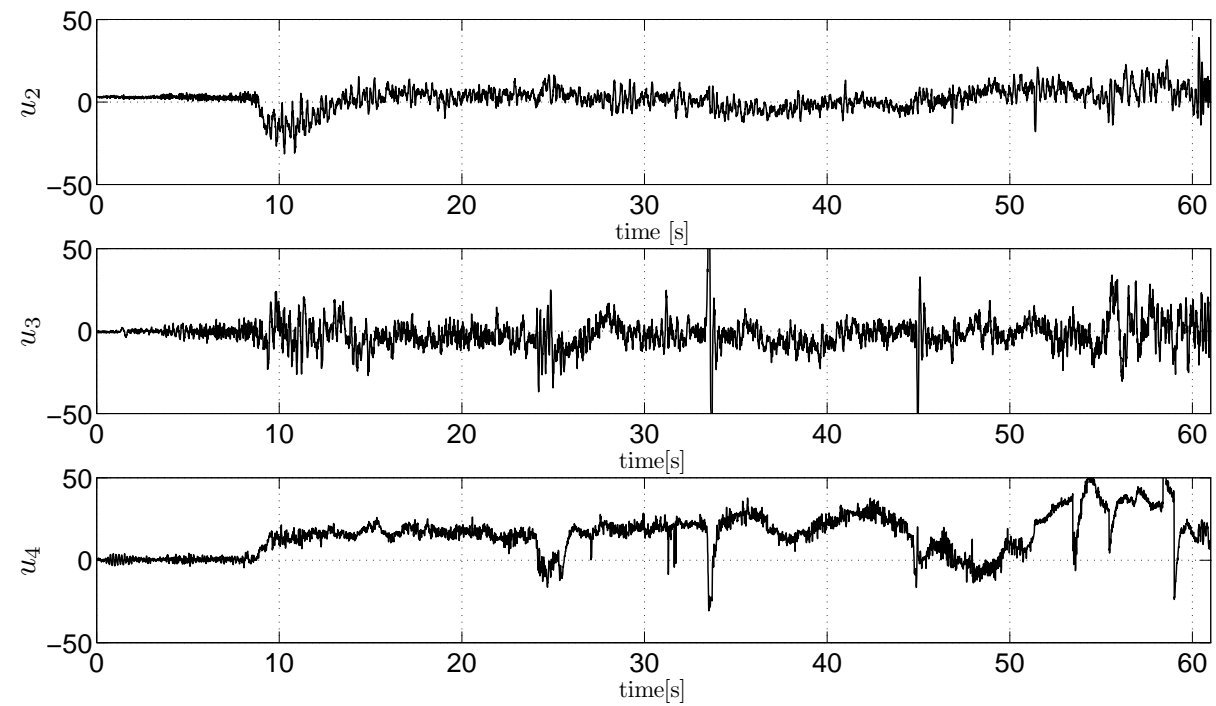

Figure 7. Control inputs $u_{2}, u_{3}$ and $u_{4}$.

${ }^{5}$ Hoffmann, G., Huang, H., Waslander, S. L., and Tomlin, C., "Quadrotor helicopter flight dynamics and control: Theory and experiment," Proc. of the AIAA Guidance, Navigation, and Control Conference, Hilton Head, South Carolina, USA, 2007.

${ }^{6}$ Hespanha, J., Yakimenko, O., Kaminer, I., and Pascoal, A., "Linear Parametrically Varying Systems with Brief Instabilities: An Application to Integrated Vision/IMU Navigation," IEEE Transactions on Aerospace and Electronic Systems, Vol. 40, No. 3, July, 2004, pp. 889-902.

${ }^{7}$ Holt, R. S. and Beard, R. W., Vision-Based Road-Following Using Proportional Navigation, Selected papers from the 

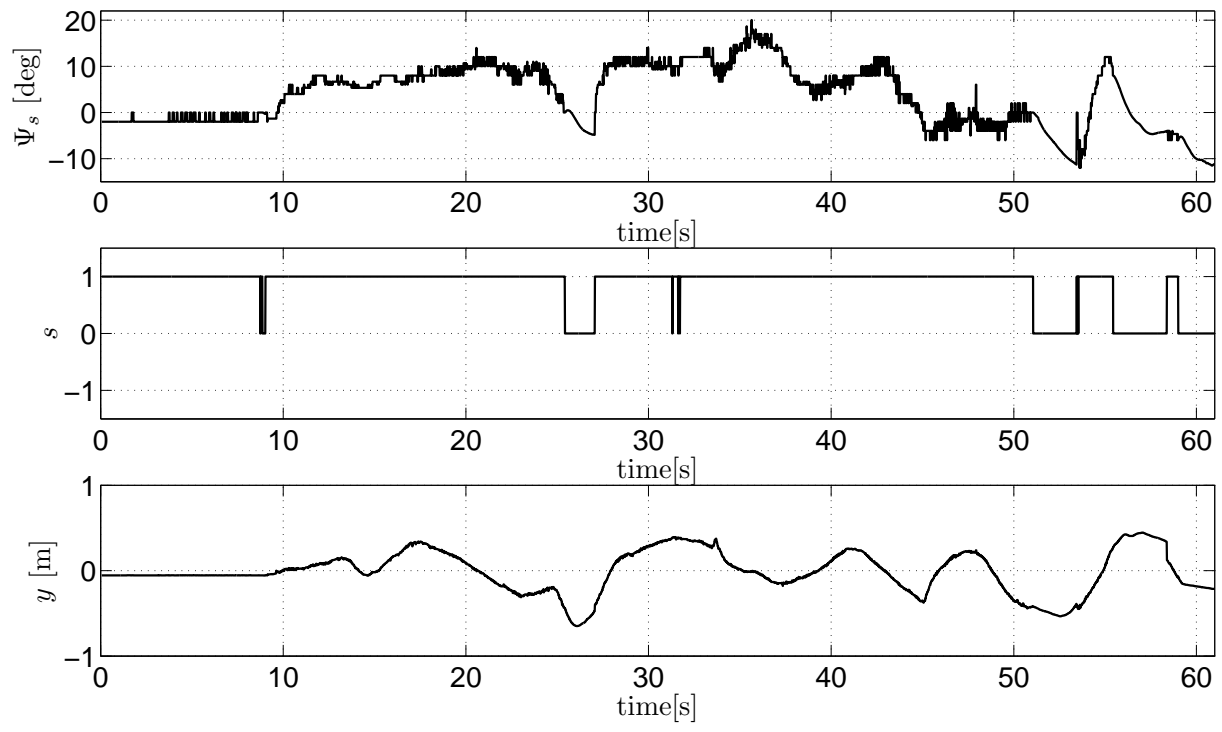

Figure 8. Switching signal $s, \psi_{s}$ and $e_{y s}$. At $t=25$ and $t=51$ the vehicle was disturbed as we can see from the angle measurement $\psi_{d}$.

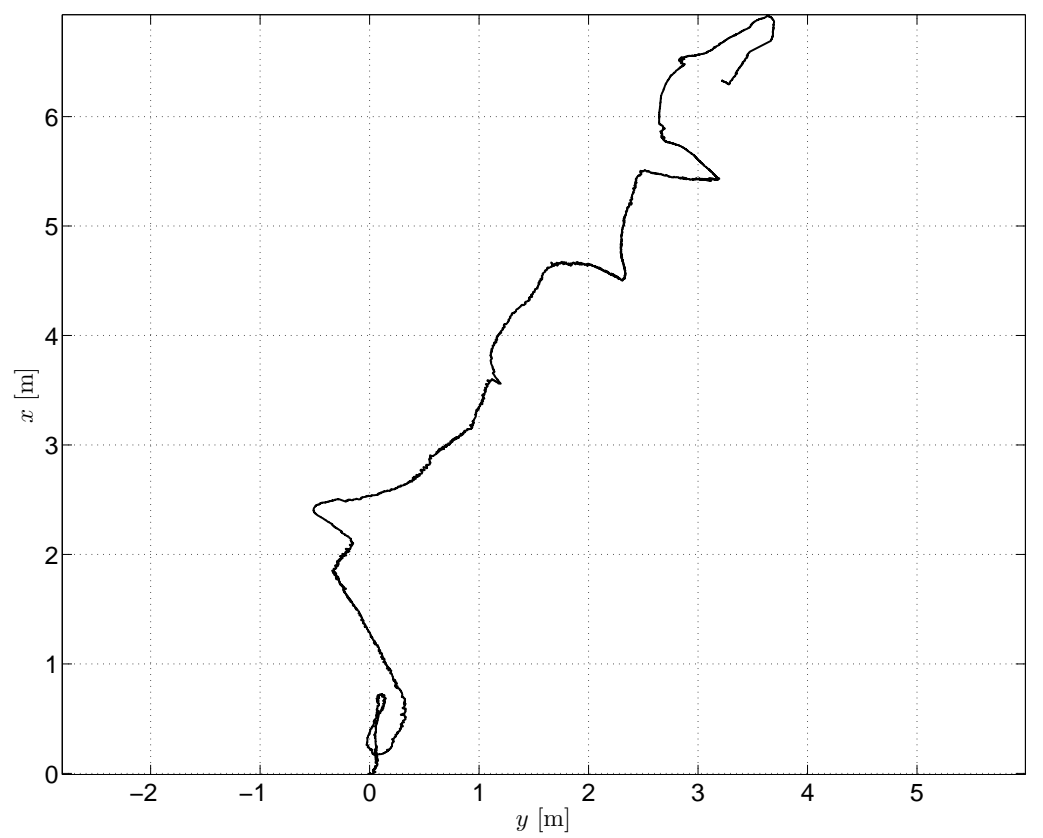

Figure 9. $x-y$ plane of the navigated path. In this figure we show the switching effect in the lateral position dynamics (see peaks in $(x, y)=(2.4,-0.55),(x, y)=(4.6,2.2)$ and $(x, y)=(5.45,3.15)$ ) when the system is disturbed and the camera losses the highway image.

2nd International Symposium on UAVs, Reno, Nevada, U.S.A. June 810, 2009, Reno, Nevada, 2009.

${ }^{8} \mathrm{Xu}$, A., Viriyasuthee, C., and Rekleitis, I., "Optimal Complete Terrain Coverage using an Unmanned Aerial Vehicle," Proc. IEEE International Conference on Robotics and Automation (ICRA2011), Shanghai, China, may 2011, pp. 2513-2519.

${ }^{9}$ Freidovich, L. B. and Khalil, H. K., "Logic-Based Switching for the Control of a Class of Nonlinear Systems," Proc. IEEE Conference on Decision and Control (CDC'2003), Maui, Hawaii USA, Dec. 2003, pp. 1059-1064.

${ }^{10}$ Freidovich, L. B. and Khalil, H. K., "Lyapunov-based switching control of nonlinear systems using high-gain observers," Automatica, Vol. 43, No. 1, 2007, pp. 150-157. 
${ }^{11}$ Bouabdallah, S. and Siegwart, R., "Backstepping and Sliding-mode Techniques Applied to an Indoor Micro Quadrotor," In Proceedings of IEEE Int. Conf. on Robotics and Automation, Barcelona, Spain, April 2005, pp. 2247-2252.

${ }^{12}$ Bouabdallah, S., Design And Control of Quadrotors with Application to Autonomous Flying, Ph.D. thesis, Ecole Polytechnique Federale De Lausanne, Lausanne, Feb. 2007.

${ }^{13}$ Chaumette, F., "Image moments: a general and useful set of features for visual servoing," IEEE Trans. on Robotics, Vol. 20, No. 4, August, 2004, pp. 713-723.

${ }^{14}$ Flores, G., Garcia, L., Sanahuja, G., and Lozano, R., "PID Switching Control for a Highway Estimation and Tracking Applied on a Convertible Mini-UAV," Proc. 51st IEEE Conference on Decision and Control (CDC'2012), Maui, HI, USA, Dec. $2012,3110-3115$

${ }^{15}$ Slotine, J. and Li, W., Applied Nonlinear Control, Prentice Hall, 1990.

${ }^{16}$ Liberzon, D., Switching in Systems and Control, Birkhuser, Boston, 2003.

${ }^{17}$ Hespanha, J. P. and Morse, A. S., "Stability of Switched Systems with Average Dwell-time," Conference on Decision and Control, December 1999, pp. 2655-2660.

${ }^{18}$ Khalil, H. K., Nonlinear Systems, Prentice Hall, New York, 2002.

${ }^{19}$ Peleties, P. and DeCarlo, R., "Asymptotic Stability of m-Switched Systems using Lyapunov-Like Functions," IEEE American Control Conference. 